\title{
APUNTES DOCUMENTALES SOBRE INMIGRANTES EUROPEOS Y NORTEAMERICANOS EN LIMA (SIGLO XIX)
}

\author{
POR \\ TEODORO HAMPE MARTINEZ \\ Institut für Geschichte \\ Universidad de Viena
}

\section{ASPECTOS GENERALES DE LA INMIGRACIÓN}

Desde los comienzos de la historia republicana del Perú, el propósito de fomentar la inmigración de gente blanca, de preferencia europea, fue manifestado constantemente por los gobernantes del país. Lo que se buscaba en dicha gente era, ante todo, el conocimiento tecnológico y los novedosos métodos de inversión capitalista que pudieran traer consigo; también se pensaba, por cierto, en la virtualidad de "regenerar" el espíritu de los peruanos - vistos como haraganes y retrasados por naturaleza- mediante la incorporación de una raza que se consideraba superior. Sobre esta base se generaron durante el siglo XIX muchas expectativas de cambio en el desarrollo económico y social de la república. En realidad, pese a que los extranjeros vinieron al Perú en corto número y beneficiaron más el crecimiento de las ciudades que la expansión rural -contrariamente a lo que habían ideado los gobernantes-, desde un principio desarrollaron estrechos vínculos con la población autóctona, asumiendo de tal manera un rol decisivo en la historia nacional.

El sentimiento de los peruanos, y especialmente de la clase alta limeña, con respecto a la incorporación de gente blanca se halla bien condensado en el libro que Juan de Arona (i.e. Pedro Paz Soldán y Unanue) publicó en 1891, La inmigración en el Perú. Esta obra critica la falta de brazos en la agricultura causada por la manumisión de los esclavos negros, elogia las virtudes de laboriosidad de los europeos y aplaude el ejemplo de masiva integración de pobladores extranjeros realizado en la Argentina». 
Juan de Arona comenta explícitamente: "La inmigración europea, que para la generalidad es cuestión de brazos, de aumento de población y de mejoramiento de la raza, se ha hecho para nosotros, después del estancamiento y aun retroceso que han sucedido a la guerra, cuestión de alta política y nada menos que de salvación nacional» (1)

Se ha observado con acierto que un estudio integral de la problemática que plantea la inmigración en el siglo XIX requiere de un enfoque bifronte, que analice tanto la coyuntura política y económica en las naciones de origen de los migrantes como las estructuras vigentes en la sociedad peruana al momento de su llegada (2). Una manera tradicional de abordar el problema ha sido examinando los diversos instrumentos legales que se promulgaron después de la independencia sobre la condición de los extranjeros en el Perú. De hecho, al principio la legislación en la materia fue extremadamente liberal, subordinada al objetivo de fomentar la permanencia de los forasteros.

La primera ley referida específicamente a la inmigración data de 17 de noviembre de 1849, durante el primer gobierno de Castilla. Señalaba esta ley que, debido al "grado de postración en que se halla la agricultura del país por falta de brazos", era necesario promover la venida de extranjeros, y para ello se ofrecía una prima de 30 pesos por cabeza a todo aquel que introdujera colonos de 10 a 40 años de edad, siempre que viniesen en grupos mayores de cincuenta individuos. Fue al amparo de dicho instrumento, dictado bajo la presión (interesada) del hacendado y político liberal Domingo Elías, que tuvo lugar la masiva importación de culíes o braceros chinos al Perú (3).

Por otra parte, la misma ley inmigratoria "chinesca» incentivó la suscripción de varios contratos del Estado con particulares, quienes se comprometieron a traer colonos de diferentes naciones de Europa. Pero casi todos estos proyectos acabaron en el fraca-

(1) Juan de Arona, La inmigración en el Perú. Monografía histórico-crítica, Lima: Imp. del Universo, 1891, pág. X. Con ocasión del sesquicentenario de la independencia nacional, esta obra fue reeditada en un volumen con varias colaboraciones titulado La inmigración en el Perí, Lima: Academia Diplomática, 1971, pág. 27-214. Dicho volumen contiene una valiosa "Bibliografía de la inmigración en el Perú", elaborada por Sylvia Stuard de la Torre (pág. 20-25).

(2) Giovanni Bonfiglio Volpe, "Introducción al estudio de la inmigración europea en el Perú", en Primer Seminario sobre Poblaciones Inmigrantes. Actas Lima: Consejo Nacional de Ciencia y Tecnología, 1988, vol. 1.

(3) ARONA " [1], págs. 3-5. Este autor celebra la importación de trabajadores chinos como "a la más larga, a la más duradera, a la más completa, a la sola inmigración verdadera del Perú", (pág. 39). 
so, como la introducción de 320 agricultores irlandeses destinados a las haciendas del doctor Juan Gallagher en el Callao (1851), la expedición de 1.150 trabajadores alemanes contratados por el empresario Antolín Rodulfo para laborar en la selva amazónica (1851) o el asentamiento de 58 familias oriundas de Guipúzcoa en los campos algodoneros de Talambo, propiedad de Manuel Salcedo (1860) (4). Unicamente logró éxito la empresa dirigida por el barón Cosme Damián Schütz, que en 1857 llevó a cerca de 300 labradores originarios de Renania y el Tirol a colonizar - en una osada aventura - la cuenca agrícola del Pozuzo, productora de arroz, azúcar, coca, café, yuca y otros frutos; once años más tarde, cuando la colonia germánica del Pozuzo era ya una feliz realidad, se unió a dicho grupo otro contingente de 300 labradores tiroleses y bávaros (5).

El gobierno civilista de Pardo se interesó también vivamente en la importación de gente europea, a fin de activar sus planes "progresistas», de desarrollo material de la república. Una de sus primeras disposiciones fue la creación de la Sociedad de Inmigración Europea, instituida formalmente en marzo de 1873, con el objeto de administrar los fondos estatales para la inmigración, celebrar los contratos respectivos, cuidar del viaje, alojamiento y ubicación de los migrantes, proveerles de animales domésticos, semillas, puestos de trabajo, etc. A manera de complemento se promulgó la ley de inmigración de 28 de abril de 1873, que autorizaba la inversión de 100.000 soles anuales para fomentar la venida de trabajadores europeos y favorecía especialmente la contrata de artesanos, obreros y domésticos de ambos sexos, que contarían con los gastos de transporte cubiertos por el Estado (6). Los efectos de la ley inmigratoria de Pardo se redujeron virtual-

(4) Mario E. DEL Río, La inmigración v su desarrollo en el Perú. Lima: Sanmartí, 1929, págs. 51-53. La expedición de los colonos guipuzcoanos a Talambo donde tuvo lugar el famoso "incidente" de 1863, es tratado entre varios por Demetrio Ramos Pérez, "Fases de la emigración española a Hispanoamérica en el siglo XIX", en Jahrbuch für Geschichte von Staat, Wirtschaft und Gesellschaft Lateinamerikas, Colonia, no 13, 1976, págs. 170-171.

(5) Karl Schmid-Tannwald, Pozuzo, vergessen im Urwald. Ein Bericht aus Peru (Braunschweig: Westermann, 1957. Véase también Manfred ILLI, Die deutsche Auswanderung nach Lateinamerika. Eine Literaturübersicht, LateinamerikaStudien, vol. 2, Munich Wilhelm Fink, 1977, que of rece una bibliografía crítica sobre la emigración alemana al Perú (págs. 128-132).

(6) DEL Rio, [4], págs. 55-56. Una declaración oficial del gobierno civilista se justificaba diciendo que "nadie puede desconocer la falta que de esa gente se hace sentir en todas partes de la república, y sobre todo en los pueblos de la costa, en donde absolutamente no hay hijo del país que quiera dedicarse al servicio de las familias y adonde es difícil encontrar un artesano" (p. 57). 
mente a la entrada de unas 3.000 personas, en su mayoría de origen italiano, que colonizaron las montañas de Chanchamayo (7).

En la época de reconstrucción nacional que siguió a la guerra con Chile continuó pensándose en las virtualidades de la mano de obra extranjera, tal como lo demuestra la ley de inmigración de 14 de octubre de 1893, expedida por el gobierno de Morales Bermúdez. Este dispositivo protege y fomenta la incorporación de migrantes de raza blanca, menores de 60 años de edad, a los cuales concede derechos de alojamiento y manutención, exoneración de impuestos, apropiación de terrenos cultivables, provisión de instrumentos de trabajo y otras jugosas ventajas. Sin embargo, al igual que las disposiciones previas relativas a la inmigración oficial - apoyada financieramente por el Estado-, dicha ley no rindió los frutos esperados (8).

En este contexto interesa hacer una distinción entre la inmigración oficial o provocada y la inmigración voluntaria o espontánea. Porque más valiosa, perdurable, ha sido en verdad la huella que dejaron los cientos de inmigrantes espontáneos que, sin contar con ayuda estatal, se establecieron en el Perú durante el siglo pasado. Ellos, en su mayoría, fijaron su residencia en Lima y otros núcleos urbanos de la costa (9). Su llegada al país obedeció a diversas razones: unos venían en desempeño de cargos militares o diplomáticos; otros, poseedores de cierto capital y experiencia en actividades empresariales, instalaban industrias $o$ abrían casas de comercio; y también había quienes, dotados de menos recursos, se ocupaban en labores campesinas o artesanales. Unidos muchos de ellos - hombres jóvenes y solteros-con mujeres de la tierra, su descendencia ha conservado el apellido extranjero, pero se encuentra entrañablemente ligada al destino histórico peruano.

El comercio era la actividad en que se ocupaba la mayor parte de los forasteros blancos, europeos y norteamericanos, residentes en Lima. Apoyados en la mentalidad capitalista traída de su patria, que contrastaba con los rasgos eminentemente

(7) Janet E. Worrall, La inmigración italiana en el Perú, 1860-1914, Lima: Instituto Italiano de Cultura, 1990.

(8) Abraham Padilla Bendezú, "Historia de la inmigración en el Perú", en Revista Peruana de Derecho Internacional, Lima, XXII 1962, págs. 57-130; véase especialmente el cap. 10, que ofrece una revisión histórica de la legislación en esta materia.

(9) Teodoro Hampe Martínez, "Inmigrantes en Lima (siglo XIX)", en El Comercio (Lima, 28 de junio de 1983), pág. A2. 
señoriales y tradicionales de la sociedad peruana, lograron con relativa facilidad salir exitosos en los negocios. Para muchos de tales individuos se cumplió efectivamente el proverbio de «hacer la América", que se traducía en incremento de su patrimonio y mejoramiento de su ubicación social. El éxito económico de los inmigrantes se puede explicar por una variedad de razones: el desdén hacia las ocupaciones manuales y comerciales imperante todavía en la mentalidad criolla, los vacíos institucionales y legales en la estructura administrativa peruana, la facilidad de acceso (por su color de piel) a las clases medias y acomodadas de las ciudades, el manejo de los últimos avances tecnológicos y la neutralidad política frente a las nefastas guerras civiles (10).

Ya un autor nacional constató en 1872 la condición privilegiada de los forasteros en el Perú: «El extranjero no solamente está colocado en igual situación que el ciudadano, sino que su condición es aún más favorecida, sus personas son más respetadas, sus bienes más considerados, y casi nunca le afectan las consecuencias funestas e inevitables de las revoluciones, que con tanta dureza recaen siempre sobre los peruanos" (11). Este autor citado, Félix Cipriano Coronel Zegarra, dedicó su libro La condición jurídica de los extranjeros en el Perú a refutar las calumnias sobre la falta de hospitalidad que se decía haber en el país contra los foráneos. Una acusación ciertamente falsa, sustentada tal vez en la debilidad que mostró el Estado al ceder ante las sucesivas reclamaciones promovidas por gobiernos de fuera, que recusaban el maltrato dado a los inmigrantes que venían de manera no voluntaria o bajo misérrimas condiciones de empleo.

No obstante que el Estado peruano se declaraba oficialmente católico en el siglo XIX, abundantes documentos de la época -inclusive los registros parroquiales- atestiguan que existió de hecho una considerable tolerancia de cultos, pues no se impidió a ninguna religión la práctica más o menos ostensible de sus ritos. Que las colonias extranjeras pudieron mantener sus confesiones tradicionales y reproducir las usanzas de sus tierras de origen, es una realidad confirmada por la formación de milicias urbanas propias y la publicación de periódicos en lenguas forá-

(10) Julia Alfaro Vallejos, "Los pescadores italianos de Chucuito", en Primer Seminario sobre Poblaciones Inmigrantes, vol. 2, págs. 141-142, donde se refiere a los motivos para el éxito éconómico de los inmigrantes europeos.

(11) Félix Cipriano C. Zegarra, La condición juridica de los extranjeros en el Perü, Santiago de Chile, Imp. de la Libertad, 1872, pág. XXV. 
neas (12). Pero los inmigrantes, impedidos de sobrevivir en completo aislamiento, tendieron también a integrarse con las comunidades urbanas de su entorno por medio de un complejo y dinámico proceso social, cuyas formas esenciales son dignas de particular atención.

\section{REGISTROS PARROQUIALES: FUENTES SOBRE LA INMIGRACIÓN}

Nuestra aproximación al problema de la inserción social de los extranjeros se circunscribe al grupo de los europeos y norteamericanos, o sea la población blanca por excelencia, que constituyó el objeto más deseado de la inmigración. Desde el punto de vista geográfico, nos centramos en la ciudad de Lima y, más particularmente, en la jurisdicción de la Parroquia del Sagrario (templo contiguo a la catedral metropolitana), que comprende todas las calles principales en el contorno de la Plaza de Armas. Una revisión de los registros sacramentales del Sagrario en el siglo XIX nos permite comprobar que se trataba de la parroquia socialmente más importante de Lima, aquella a la cual pertenecían los ricos comerciantes asentados en el jirón de la Unión, los profesionales más prestigiosos y los dueños de casonas solariegas heredadas del coloniaje.

La consulta de los libros parroquiales de bautismos, matrimonios y defunciones revela a estos instrumentos como fuentes sumamente provechosas para seguir el rastro de los extranjeros que se afincaron en la capital peruana. A lo largo del primer siglo de vida republicana, hemos ubicado más de trescientos documentos sacramentales que se refieren a inmigrantes europeos o norteamericanos y sus descendientes inmediatos (13). Tales documentos no sólo registran los datos propios de cada bautizo, casamiento o muerte, sino también ayudan a determinar lugares

(12) Brenda Harriman, "Los británicos en el Perú", en Primer Seminario sobre Poblaciones. Immigrantes, [2], vol. 2, pág. 154, describe el funcionamiento de la guardia urbana británica y la aparición del Callao and Lima (jazette, en 1871. Por su parte Jorge BASADRe, en Historia de la Repuiblica del Perí, Lima, 1961, tomo I, pág. 187, sostiene que "Las reiteradas disposiciones constitucionales, legales y administrativas para asimilar a los extranjeros útiles al quehacer nacional dentro de la comunidad peruana quedaron, como tantas otras de la época, en el plano de la teoría. Sobre todo en relación con el comercio, se fueron formando y desarrollando las colonias de súbditos de otros paises, ajenas a esta comunidad".

(13) He revisado los archivos de la Parroquia del Sagrario gracias a la permisión del antiguo párroco, Mons. Zacarías Untiveros (jonzálè. 
de procedencia, fecha y circunstancias de arribo, confesión religiosa y modo de integración al ambiente social limeño de los diversos migrantes. Según habrá ocasión de mostrarlo enseguida, las partidas con mayor riqueza de información para una historia social (y también para la genealogía) son las bautismales y matrimoniales.

Desde los años anteriores a la proclamación de la independencia aparecen conversiones al catolicismo de hombres adolescentes y adultos, europeos que provenían de religiones como la luterana, anglicana, presbiteriana u ortodoxa. Este es el caso del inglés Boler, de 16 años de edad (1810), el ucraniano Rachitoff, de 17 años (1901), el alemán Kast, de 24 años (1854), el escocés Hague, de 28 años (1850), el alemán Kruger, de 29 años (1859), y el también alemán Roedel, de 32 años (1868), entre otros. Hubo además varios inmigrantes no católicos - germanos y británicosque tomaron las aguas bautismales para contraer inmediatamente matrimonio con una mujer peruana. Así se comportaron en efecto los luteranos Nobelich (1833), Pflücker (1836), Lembcke (1836) y Solf (1851), y más tarde los anglicanos Hemmerde (1884) y Gubbins (1891).

No faltan desde luego referencias a algunos individuos no católicos que mantuvieron su credo protestante a pesar de una larga residencia en Lima y de haber tomado como esposas a mujeres lugareñas; la restringida tolerancia de cultos de la época exigía, sin embargo, que los hijos de matrimonios mixtos fueran educados conforme a la doctrina católica. Por otra parte, es interesante contemplar la situación de los inmigrantes que se unieron en matrimonio sólo después de una prolongada permanencia en la ciudad, como si estuvieran aguardando a consolidar su patrimonio antes de formar una familia. Entre los residentes de larga estadía podemos mencionar al parisino Courrejolles, que casó luego de 20 años (1845); el hamburgués George, luego de 19 años (1839); el girondino Grellaud, luego de 16 años (1851); el lotaringio Kruger, luego de 14 años (1843); el londinense Winder, luego de 12 años (1833), etc.

Más importante es fijarse en el origen social de las parejas con quienes los migrantes extranjeros formaron hogar en la capital peruana. Hubo un puñado de afortunados burócratas, diplomáticos y militares que tomaron la mano de hijas de la nobleza colonial limeña, pertenecientes a familias como los Aliaga, Lavalle, Moreyra, Ramírez de Arellano y Manrique de Lara. Otros 
inmigrantes debieron conformarse con desposar a las hijas de distinguidos magistrados y líderes políticos del período de la independencia, como Méndez Lachica, Mariátegui y López Aldana. Esta casta privilegiada incluye, en el lado de los inmigrantes, a varios personajes que con su lucido matrimonio marcaron la fundación de importantes linajes de la era republicana: Osma (1810), Gambini (1813), Kelly (1827), Radcliff (1830), Browne (1831), Soyer (1833), Prevost (1841), Swayne (1852), Bryce (1854).

En el otro extremo de la pirámide social se ubican los advenedizos europeos y norteamericanos que celebraron enlace con humildes migrantes provincianas y mujeres bastardas (o hijas de padres desconocidos). La lista en este grupo es bastante extensa, por lo que sólo cabe mencionar unos cuantos ejemplos, poniendo el énfasis en la procedencia de las esposas. A la serie corresponden el italiano Venzano, casado con piurana (1811), el irlandés Conroy, casado con ayacuchana (1827), el espanol Umbert, casado con huanuqueña (1830), el inglés Brandon, casado con tacneña (1846), el italiano Baglietto, casado con paiteña (1847), el francés Baumann de Metz, casado con chancaína (1850), el suizo Broggi, casado con trujillana (1853), el italiano Solari, casado con iqueña (1853), el alemán Nohesell, casado con arequipeña (1856), el alemán Gildemeister, casado con lambayecana (1869), etc.

Además, en varias oportunidades se registran casamientos de los extranjeros con mujeres sudamericanas oriundas de países vecinos al Perú; así se encuentran esposas procedentes de Quito, Guayaquil, Santiago de Chile y Tarija. Desde mediados del siglo XIX constituye un fenómeno notable la aparición de mujeres europeas en Lima, venidas formando parte de las grandes expediciones colonizadoras o como hijas de forasteros que se radicaron en la capital. La serie de matrimonios entre inmigrantes europeos en la Parroquia del Sagrario empieza con la unión del genovés Domingo Vallarino y su paisana Eugenia Maynetto, en 1847, y es continuada con los enlaces de otras doncellas italianas (Suito, Canevaro, Merea, Musso, Corvetto, Rivarola), francesas (Albertini, Othéguy, Bastard, Baserre, Perron), españolas (Fleischmann, Casanova), inglesas (Markholz) y suizas (Luzzani).

Caso singular es el que representa Tomás Garvasi, súbdito italiano, quien en 1877 contrajo nupcias con una sirvienta china llamada Juana Denegri. Como era usual por entonces, la migrante asiática había adoptado con el bautizo el apellido de la familia donde trabajaba (en este caso, el matrimonio italo-peruano 
Camogli-Denegri) (14). El hecho demuestra claramente que no todos los extranjeros lograron "hacer la América», enriqueciéndose y ascendiendo de categoría social; además, estaban descargados de los prejuicios raciales contra los chinos que afectaban a la mayoría de peruanos.

Por último mencionaremos a las hijas de residentes foráneos que formaron hogar con nuevos inmigrantes venidos de los mismos países de origen en Europa, contribuyendo de tal modo al fortalecimiento de las colonias extranjeras en Lima. Por ejemplo, tres hijas del italiano Valega casaron con sus compatriotas Denegri (ca. 1832), Dinegro (1833) y Canevaro (1834); dos hijas del francés Larrabure casaron con sus compatriotas Carriquiry (1884) y Truel (1891); una hija del inglés Guise casó con el irlandés Dartnell (1848); una hija del irlandés Conroy casó con el inglés Jameson (1850); una hija del vizcaíno Andraca casó con su compatriota Igartua (ca. 1860); una hija del alemán Pflücker casó con su compatriota Sattler (1863); una hija del italiano Canevaro casó con su compatriota Migliorati (1864), y otras más por el estilo.

La Tabla 1 resume estadísticamente, por grupos nacionales de migrantes, la información de 210 partidas de bautismos, matrimonios y defunciones pertenecientes a la Parroquia del Sagrario de Lima. Todas estas partidas conciernen directamente a la presencia de europeos y norteamericanos dentro del ambiente social limeño, desde 1810 hasta 1915; no se han considerado aquí los documentos relativos a sus hijos o parientes políticos. Como se deja notar en la tabla, la mayoría de los extranjeros avecindados en el centro de la ciudad estuvo formada por hombres procedentes de Alemania, Francia e Italia, grupos nacionales que suman algo más de $60 \%$ de la población investigada en este trabajo. Siguen en orden cuantitativo los inmigrantes de las islas británicas (ingleses, escoceses e irlandeses), que alcanzan en conjunto 16,2 \%, y después los de Norteamérica, con 6,7\%.

(14) La boda, celebrada el 22 de agosto de 1877, tuvo como padrinos a Luis Camogli y Petronila Denegri de Camogli. Véase en el Registro de bautismos, matrimonios y defunciones, inf ra, $\mathrm{n}^{-} 159$. 
TABLA 1. BAUTISMOS, MATRIMONIOS Y DEFUNCIONES EN LA PARROQUIA DEL SAGRARIO DE LIMA (1810-1915).

\begin{tabular}{|c|c|c|c|c|c|}
\hline & $\mathrm{BAU}$ & MAT & DEF & TOTAL & INDICE \\
\hline 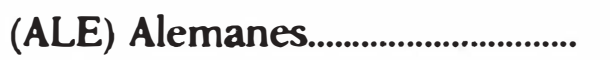 & 9 & 26 & 5 & 40 & 19,05 \\
\hline 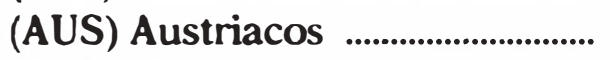 & - & 1 & - & 1 & 0,48 \\
\hline 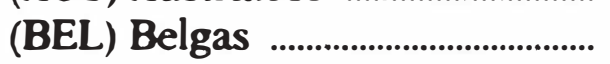 & 1 & 1 & - & 2 & 0,95 \\
\hline 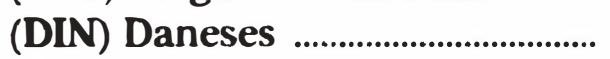 & · - & 4 & - & 4 & 1,90 \\
\hline 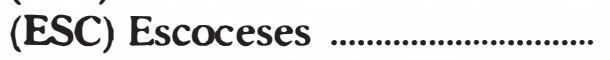 & 2 & 3 & 1 & 6 & 2,86 \\
\hline (ESP) Españoles & - & 9 & 1 & 10 & 4,76 \\
\hline 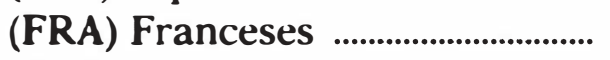 & - & 33 & 11 & 44 & 20,95 \\
\hline 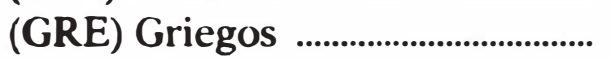 & - & 1 & - & 1 & 0,48 \\
\hline 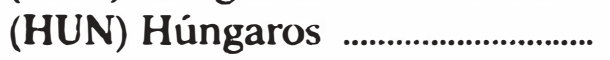 & - & 1 & - & 1 & 0,48 \\
\hline 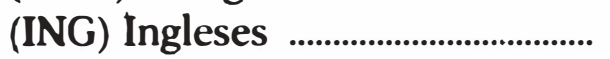 & 2 & 11 & 4 & 17 & 8,10 \\
\hline 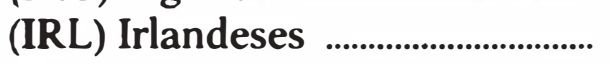 & 1 & 8 & 2 & 11 & 5,24 \\
\hline 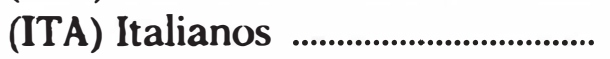 & - & 38 & 5 & 43 & 20,48 \\
\hline (POL) Polacos & - & 2 & 1 & 3 & 1,43 \\
\hline (POR) Portugueses ............................. & - & 1 & - & 1 & 0,48 \\
\hline 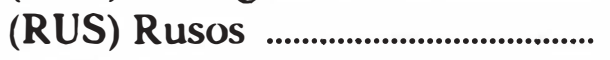 & 1 & - & - & 1 & 0,48 \\
\hline 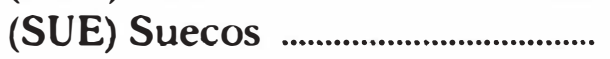 & - & 4 & 1 & 5 & 2,38 \\
\hline 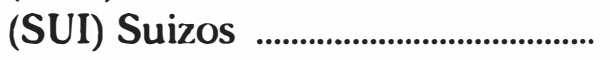 & - & 4 & 2 & 6 & 2,86 \\
\hline 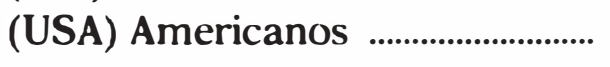 & - & 10 & 4 & 14 & 6,67 \\
\hline TOTAL & 16 & 157 & 37 & 210 & 100,00 \\
\hline
\end{tabular}

El Registro de bautismos, matrimonios y defunciones, expuesto a continuación, contiene sumillas de cada uno de los documentos interesantes a nuestro tema que han sido localizados en los archivos de la Parroquia del Sagrario. Se incluyen 16 partidas de bautismos, 37 de defunciones y 157 de matrimonios, comprendiendo una serie de referencias colocadas al final acerca de parejas que tuvieron su hogar en la jurisdicción parroquial del Sagrario, pero de las cuales no se conservan datos exactos sobre su casamiento. Con el objeto de ayudar a la futura investigación y facilitar la consulta de este registro, se anota en cada ficha - hasta donde ha sido posible - la ubicación exacta del documento (folio y libro dentro de las respectivas series de bautismos, matrimonios o defunciones) y se agrega en las últimas páginas un índice de los apellidos extranjeros. Así queda conformado un elenco, ciertamente provisional, de los aventurados y emprendedores forasteros que adoptaron como morada la ciudad de Lima durante el siglo XIX. 


\section{REGISTRO DE BAUTISMOS, MATRIMONIOS Y DEFUNCIONES \\ DE LA PARROQUIA DEL SAGRARIO DE LIMA (1810-1915)}

001/ MAT. 03-AGO-1810.

Dr. Gaspar Antonio de Osma, alcalde del crimen de la Real Audiencia, natural de Nalda (Calahorra), hijo de Joaquín de Osma y Morera y Maria Balbanera de Tricio y Querejazu (ESP), casado con María Josefa Rosa Ramírez de Arellano, natural de Lima, hija del coronel Domingo Ramírez de Arellano y Catalina Baquijano y Carrillo. /Lib. 11, f. 174v/

002/ BAU. 21-DIC-1810.

José Guillermo Boler, inglés, de 16 años, hijo de Domingo y Mercedes Boler (ING), apadrinado por José Bernardo de Tagle, marqués de Torre Tagle, comisario de guerra del virreinato. / Lib. 17, f. 421 /

003/ MAT. 24-FEB-1811

Antonio María Sacio, natural de Gurra (Génova), hijo de Nicolás Sacio y Maria Magdalena Boragno (ITA), casado con Maria Gertrudis Poleo, natural de Lima, hija de Bartolomé Poleo y Josefa Nieto. /Lib. 11, f. 180/

004/ MAT. 22-DIC-1811

Félix Valega, natural de Quiliano (Savona), hijo de Juan Bautista Valega y María Lanza (ITA), casado con Maria Josefa Iribar, natural de Lima, hija de Pablo Iribar y Manuela Espinoza. /Lib. 11, f. 186v/

005/ MAT. 22-DIC-1811

Antonio Venzano, natural de Sestri (Génova), hijo de Juan Bautista Venzano y María Nicolasa Dagnino (ITA), casado con Andrea Bayeto, natural de Piura, hija de Juan Bayeto y Ana Romero. /Lib. 11, f. 187/

006/ MAT. 20-ENE-1813

José Gambini, natural de Génova, hijo de Antonio Gambini y Ana Sermengo (ITA), casado con Rosa Manrique de Lara, natural de Lima, hija de Felipe Sixto Manrique de Lara y Lucía Calle y Hurtado. /Lib. 11, f. 190v/

007/ MAT. 11-DIC-1820

Antonio Maria Sacio, natural de Gurra (Génova), viudo de Marla Gertrudis Poleo (ITA), casado con Juana Moreno, natural de Lima, hija de Nicolás Moreno y Josefa Tejada. /Lib. 11, f. 292v/

008/ MAT. 28-OCT-1824

Antonio de Sousa Ferreyra, natural de Vilanova de Famaliçao (Oporto), hijo de José Joaquín de Sousa Ferreyra y María Felicia Pinheiro de Alfonseca (POR), casado con Paula Gutiérrez de Caviedes, natural de Lima, hija de Jerónimo Gutiérrez de Caviedes y Rosa Arnao. /Lib. 11, f. 294v/

009/ MAT. 10-JUL-1825

Juan Branch, francés, hijo de Claudio Branch y Juana Renar (FRA), casado con 
Rosa Santander, natural de Guayaquil, viuda de Fernando Barrantes, hija de Pedro Santander y Ana de la Peña. / Lib. 12, f. 1 v/

010/ MAT. 28-JUL-1825

Manuel Ricardo Smith, natural de Londres, hijo de Juan y Maria Smith (ING), casado con Petronila Panizo, natural de Lima, hija de Juan Panizo y María Dolores Talamantes. /Lib. 12, f. 5v/

011/ MAT. 21-NOV-1825

Julio Rousson, natural de Burdeos, hijo de Juan e Isabel Rousson (FRA), casado con Teresa Cuadra, viuda de Juan de Dios Rivero. /Lib. 12, f. 6v/

012/ MAT. 02-ENE-1827

Patricio Guillermo Kelly, natural de Roscommon, hijo de Guillermo Kelly y Juana Lafan (IRL), casado con Rosa María de Aliaga, natural de Lima, hija de Juan de Aliaga y Colmenares, conde de San Juan de Lurigancho, y Juana Calatayud y Bolaños. /Lib. 12, f. 25/

013/ MAT. 18-FEB-1827

El general Carlos Luis del Gillo, griego, viudo de Margarita Giglis (GRE), casado con Micaela Ortiz de la Daga, natural de Lima, hija de Juan Manuel Ortiz de la Daga y María Santos Gayo. /Lib. 12, f. 69/

014/ MAT. 25-MAR-1827

Domingo Ginocchio, natural de Génova, hijo de Domingo Ginocchio y Magdalena Blanco (ITA), casado con María del Rosario Godomar, natural de Santiago de Chile, hija de Andrés Godomar y Trinidad Villarroel. /Lib. 12, f. 30v/

\section{5/ MAT. 12-MAY-1827}

El vicealmirante Martín Jorge Guise, natural de Highoasis Court (Gloucestershire), hijo de sir Juan Guise y María Isabel Wright (ING), casado con Juana María del Valle y Riestra, hija de Antonio del Valle e Isabel de la Riestra. /Lib. 12, f. 54v/

016/ MAT. 17-MAY-1827

Sebastián Massa, natural de Malere, viudo de Catalina Amador (ITA), casado con Bernardina Valenzuela, natural de Lima, viuda de José Dulanto. /Lib. 12, f. 26/

017/ MAT. 04-OCT-1827

Santiago Risso, natural de Génova, hijo de Francisco Risso y María Colomba Carbona (ITA), casado con Andrea Fernández, natural de Lima, hija de Antonio Fernández y Bernarda Masa. /Lib. 12, f. 41v/

018/ MAT. 22-DIC-1827

Tomás Conroy, natural de Wexford, hijo de Tomás Conroy y Cristina Herring (IRL), casado con Petronila Paula Enderica, natural de Ayacucho, hija de Miguel Enderica y Rosa María Talamantes. /Lib. 12, f. 40/

019/ MAT. 01-ENE-1829

Estanislao Pedro Osterling, natural de Estocolmo, hijo de Pedro Osterling y María 
Olofisdotter ( $S U E$ ), casado con Angela Zavala, natural de Lima, hija de (jabriel Zavala y María Nieve's Jiménez. /Lib. 12, f. 62v/

020/ DEF. 28-ENE-1829

El vicealmirante Martín Jorge Guise, natural de Highoasis Court (Gloucestershire), marido de Juana María del Valle y Riestra (ING), enterrado en la Catedral.

021/ BAU. 12-MAY-1830

Arnaldo Oboussier, natural de Amberes, hijo de Félix Oboussier y María Luisa Amy (BEL), apadrinado por Francisco Quirós. /Lib. 20, f. 11 /

022/ MAT. 24-JUL-1830

Alejandro Hamilton Radcliff, natural de Nueva York, hijo de Guillermo y Catalina Radcliff (USA), casado con Rosa Méndez, natural de Lima, hija del Dr. Pedro José Méndez. Lachica y Manuela Martínez. /Lib. 12, f. 92 /

023/ MAT. 06-SET-1830

Gabriel Umbert, natural de Mahón (Menorca), hijo de Gabriel Umbert y María Vila (ESP), casado con María Josefa Antonia Ninia, natural de Huánuco, residente en Lima desde pequeña, viuda de José Gutiérrez. /Lib. 12, f. 97v/

024/ MAT. 23-ABR-1831

Bartolomé Jorge Browne, natural de Londres, hijo de Bartolomé Browne y ... Harvey (ING), casado con María Manuela de Aliaga, natural de Lima, hija de Juan de Aliaga y Colmenares, conde de San Juan de Lurigancho, y Juana Calatayud y Bolaños. /Lib. 14, f. 187/

025/ MAT. 10-FEB-1833

El coronel Salvador Soyer, natural de Bayona, hijo de Martin Soyer y Susana Boyer (FRA), casado con Mercedes Lavalle, natural de Lima, hija de Simón Lavalle e Isabel Cavero. /Llb. 13, f. 97/

026/ BAU. 03-ABR-1833

Antonio Nobelich, natural de Neukirch (Prusia), hijo de Antonio Nobelich y Juana Scharder (ALE), apadrinado por Juan Anzardo. /Lib. 20, f. 191 v/

027/ MAT. 15-ABR-1833

Antonio Nobelich, natural de Neukirch (Prusia) ( $A L E$ ), casado con Isabel Parrau, natural del Callao, residente en Lima hace 10 años, hija de Gonzalo Parrau y Antonia Bardales. /Lib. 12, f. 153/

028/ MAT. 17-AGO-1833

Joaquín Esteban Daniel MacKay, natural de Glasgow, residente en Lima hace más de 8 años, hijo de Juan MacKay y María Campbell (ESC), casado con Tomasa Villegas, natural de Lima, hija de José Villegas y Rosa Aparicio. /Lib. 12, f. $158 \mathrm{v} /$

029/ MAT. 11-SET-1833

Pedro Dinegro, natural de Génova, residente en Lima hace 1 año, hijo de José 
Dinegro y Emilia Asarta (ITA), casado con Isabel Valega, natural de Lima, hija de Félix Valega y Maria Josefa Iribar. /Lib. 12, f. 159v/

030/ MAT. 21-OCT-1833

Miguel Francisco Winder, natural de Londres, residente en Lima hace 12 años, hijo de Miguel Winder y Ana Williamson (ING), casado con Carmen Lorca, natural de Lima, hija de Juan Lorca y Toribia Bueno. /Lib. 12, f. 161/

031 / MAT. 29-ABR-1834

José Francisco Canevaro, natural de Zoagli (Génova), hijo de Jacobo Canevaro y Batistina Raggio (ITA), casado con Francisca Zenona Valega, natural de Lima, hija de Félix Valega y María Josefa Iribar. /Lib. 12, f. 166/

032/ MAT. 16-JUN-1834

Francisco Saberbein, natural de Pest, residente en Lima hace 2 años, hijo de Vltorino Saberbein y Margarita Sehirmin (HUN), casado con Angela Villavicencio, natural de Lima, hija de Carlos Vlllavicencio y Rosa Bartola Guzmán. /Lib. 12, f. $168 /$

\section{3/ MAT. 17-NOV-1834}

Federico Augusto Matías Elmore, natural de Londres, hijo de Enrique Matías Elmore y Ana Polhill Percy (ING), casado con Josefa Fernández de Córdova, natural de Quito, hija del Dr. Diego Fernández de Córdova e Isidora Alméstar. /Lib. 14, f. 252v/

\section{4/ MAT. 24-DIC-1834}

Roberto Polack, natural de Milán, residente en Lima hace 6 años, hijo de Ignacio Polack y María Fuente (ITA), casado con Josefa Dávalos, natural de Quito, residente en Lima hace más de 10 años, hija de Mariano Dávalos y Rosa Pastrana. /Lib. 12, f. 173v/

\section{5/ MAT. 29-MAY-1836}

Juan Ignacio Flint, natural de Charleston (Carolina del Sur), residente en Lima hace 10 años, viudo de Beatriz. Alcázar (USA), casado con María del Carmen Alcázar, natural de Lima, hija de Ignacio Antonio Alcázar y Feliciana Barrón. /Lib. 12, f. 192/

036/ MAT. 01-JUL-1836

Miguel Montané, natural de Beaumont-de-Lomagne (Tarn-et-Garonne), hijo de Francisco Montané y Luisa Lubor (FRA), casado con Jacoba Brignardelli, natural de Lima, hija de Juan Brignardelli y Manuela Mariño. /Lib. 12, f. 193/

037/ MAT. 03-SET-1836

El general Juan O'Brien, natural de Battingloss (IRL), casado con Manuela de Herrán, hija de Alejandro de Herrán y Rosa León. / Lib. 12, f. 194/

038/ BAU. 12-OCT-1836

Julio Arnaldo Pflücker, natural de Waldenburg (Silesia), de 26 años, hijo de Bernardo Pflücker y Leonora Schmiedel $(A L E)$ ), apadrinado por Francisco Antonio Manrique (o Taramona). /Lib. 20, f. 376v/ 
039/ MAT. 15-OCT-1836

Julio Arnaldo Pflücker, natural de Waldenburg (Silesia) (ALE), casado con María Manrique (o Taramona), natural de Lima, hija de Francisco Antonio Manrique (o Taramona) y Francisca Pro. / Lib. 12, f. 203/

040/ BAU. 22-OCT-1836

Juan Federico Lembcke, natural de Hamburgo, de 26 años, hijo de Jorge Federico Lembcke y Cristina Catalina Juana Lorentzen ( $A L E)$, apadrinado por Luis Bellodas Iturrizaga. / Lib. 20, f. 376/

041/ MAT. 29-OCT-1836

Juan Federico Lembcke, natural de Hamburgo ( $A L E$ ), casado con Isabel Fabiana Bellodas, natural de Lunahuaná (Cañete), hija de Luis Bellodas Iturrizaga y Manuela Salas. / Lib. 12, f. 198v/

042/ MAT. 26-FEB-1838

Manuel José Guillermo Walden, natural de Londres, hijo de Guillermo Walden y Elena Sullevan (ING), casado con Juana Hoyos, natural de Lima, hija de Fernando Hoyos y Josefa Vilchez. /Lib. 12, f. 215v/

043/ MAT. 26-OCT-1838

Juan Guichard, natural de Burdeos, hijo de Guillermo Guichard y Juana Dutast $(F R A)$, casado con Manuela Amigo, natural de Lima, hija de Francisco Amigo y Manuela García. /Lib. 12, f. 229/

044/ MAT. 29-AGO-1839

Juan Ricardo George, natural de Hamburgo, residente en Lima hace 19 años, hijo de Ricardo e Isabel George $(A L E)$, casado con Antonia Sáenz de Tejada, natural de Lima, hija de Antonio Sáenz de Tejada y Josefa de la Cuadra. /Lib. 12, f. $241 /$

045/ MAT. 10-OCT-1839

Agustín Dorival, francés, hijo de Pedro y Teresa Dorival (FRA), casado in articulo mortis con Maria Rosas, hija de Manuel Rosas y Manuela Núñez. /Lib. 12, f. $242 v /$

046/ MAT. 22-AGO-1840

José Suito, natural de Génova, residente en Lima desde pequeño, hijo de Domingo Suito y Juana Liberti (ITA), casado con Josefa Florentina Díaz, natural de Lima, hija de José Díaz y María Torres. /Lib. 12, f. 255/

047/ MAT. 19-MAY-1841

Samuel Stanhope Prevost, cónsul de los Estados Unidos, hijo de Juan Prevost y Francisca Carolina Smith (USA), casado con María Mauricia Moreyra, hija de Francisco Moreyra y Matute y Mariana de Avellaluerte. / Lib. 12, 1. 269v/

048/ DEF. 11-AGO-1841

Antonio María Sacio, natural de Gurra (Génova), marido de Toribia Poleo (ITA), enterrado en la iglesia de San Francisco. 
049/ MAT. 01-DIC-1841

Lorenzo Deplace, natural de Samoens (Faucigny), residente en Lima hace 7 años, viudo de María Mendoza, hijo de José Deplace y Josefa Reynaud (FRA), casado con Francisca Rosas, natural de Lima, hija de Manuel Rosas y Manuela Núnez. /Lib. 11, f. 307v/

\section{0/ MAT. 24-AGO-1842}

Santiago Migone, natural de Génova, hijo de Bartolomé Migone y Blanca Borro (ITA), casado con Francisca Jacobs, natural de Lima, viuda de Antonio Pardo, hija de Melchor Jacobs e Isabel Castello. /Lib. 11, f. 318v/

\section{1/ MAT. 12-SET-1842}

Rollin Thorne, natural de Nueva York, viudo de Paula Palma, hijo de Enrique Thorne y Margarita Maguellas (USA), casado con Manuela Izaguirre, natural de Lima, hija -ilegitima - de José Izaguirre y Eusebia Lamarca. /Lib. 11, f. 328/

052/ MAT. 02-ABR-1843

Juan Bautista Tenaud, natural de Nantes, hijo de Juan Bautista Tenaud y Julia Lavire (FRA), casado con Manuela Flores, natural de Arequipa, hija de Manuel Flores y Petronila Tristán. /Lib. 13, f. 4

053/ MAT. 02-JUL-1843

Enrique D’Brot, natural de Lorient (Morbihan), residente en Lima hace 17 años, viudo de María Antonia Flores, hijo de Enrique Daniel D'Brot y Petrina Lecore (FRA), casado con Carmen Cárdenas, natural de Lima, hija de Manuel Cárdenas y Agustina Mayorga. /Lib. 11, f. 33 1v/

054/ MAT. 14-AGO-1843

Mateo Stuber, natural de Ludwigsburg (Wurtemberg), hijo de Juan Stuber y Catalina Breuntens ( $A L E$ ), casado con Francisca Barbieri, hija de Francisco Barbieri y Josefa Lascano. /Lib. 11, f. 333v/

\section{5/ MAT. 20-NOV-1843}

Juan Federico Kruger, natural de Metz (Lorena), residente en Lima hace 14 años, hijo de Martin Kruger y Mariana Humber (FRA), casado con Manuela Ruiz, natural de Lima, hija de Tomás Ruiz y Felipa Acosta. /Lib. 11, f. 337/

056/ DEF. 11-DIC-1843

Alejandro Hamilton Radcliff, natural de Nueva York, marido de Rosa Méndez (USA), enterrado en la iglesia de San Francisco.

057/ MAT. 09-DIC-1844

Sebastián Lorente, natural de Alcantarilla (Murcia), hijo de Sebastián Lorente y Juana Josefa Ibáñez (ESP), casado con Rosa Benel, hija de Pedro Benel y Josefa Borda. /Lib. 11, f. 353/

\section{8/ DEF. 20-ENE-1845}

Angel Rouaud, natural de Nantes, marido de Josefa Paz Soldán (FRA), enterrado en el Sagrario.

\section{R. I., 1993, $\mathrm{n}^{2} 198$}


059/ MAT. 30-ENE-1845

Santiago Magill, natural de Belfast, residente en Lima hace 10 meses, hijo de Hugo y Catalina Magill (IRL), casado con Juana Molero, natural de Lima, viuda de David Thornbey. /Lib. 11, f. 354/

060/ MAT. 17-NOV-1845

Pablo Francisco Ernesto Courrejolles, natural de París, residente en Lima hace 20 años, hijo de Pablo e Isabel Aurora Courrejolles (FRA), casado con Josefa Viña, natural de Lima, hija de José Viña y María López. /Lib. 14, f. 100/

061/ MAT. 23-FEB- 1846

Santiago Lanfranco, natural de Gibraltar, residente en Lima hace 5 años, hijo de Juan Lanfranco y María Rombau (ESP), casado con Francisca Tristán, natural de Arequipa, hija del general Pio Tristán y Joaquina Flores. /Lib. 11, f. 370v/

062/ MAT. 05-AGO-1846

Gilberto Brandon, natural de Londres, hijo de Josué Juan Brandon y Jesse Simonos (ING), casado con Delmira Julio Rospigliosi, natural de Tacna, hija del Dr. José Julio Rospigliosi y Antonia Murga. /Lib. 13, f. 3v/

063/ MAT. 28-NOV-1846

Agustín Bracklow, natural de Hutten, residente en Lima hace 9 años, hijo de Jacobo Bracklow y María Catalina Reja (DIN), casado con Mercedes Manrique, natural de Arequipa, hija de José María Manrique y Petronila Concha. /Lib. 13, f. $15 v /$

064/ MAT. 19-AGO-1847

Lorenzo Baglietto, natural de Génova (ITA), casado con Maria del Carmen Fornier, natural de Paita. / Lib. 13, f. 33v/

065/ MAT. 07-SET- 1847

Domingo Vallarino, natural de Génova, hijo de Tomás Vallarino y Catalina Belott (ITA), casado con Eugenia Mainetto, natural de Génova, hija de Cayetano Mainet to y Tomasa Pedevilla. /Lib. 13, f. 36/

066/ MAT. 14-SET-1847

Pedro José Himpseen, natural de Bruttig (Renania), hijo de Francisco y Margarita Himpseen $(A L E)$, casado con Juana María Ana Fleischmann, natural de Málaga, hija de José Fleischmann y Maria Antonia Berchner. /Lib. 13, f. 36v/

067/ MAT. 28-DIC-1847

Estanislao Pedro Osterling, natural de Estocolmo, viudo de Angela Zavala (SUE), casado con Josefa Clemencia Egula, natural de Lima, hija de Hilario Eguía y María Ruiz. Pimentel. /Lib. 13, f. 54/

068/ MAT. 01-JUN-1848

Juan Dartnell, natural de Newcastle (Down), residente en Lima hace 5 años, hijo de Eduardo Russell Dartnell y María Loder (IRL), casado con Mercedes Carlota 
Guise, natural de Lima, hija del vicealmirante Martin Jorge Guise y Juana María del Valle y Riestra. /Lib. 13, f. 62v/

069/ MAT. 03-JUN-1848

Enrique Ginocchio, natural de Génova, residente en Lima hace más de 3 años, hijo de Pablo Ginocchio y Catalina Bernaza (ITA), casado con Luisa Suito, natural de Génova, residente en Lima hace 7 meses, hija de Domingo Suito y Juana Liberti. /Lib. 13, f. 54/

\section{0/ MAT. 24-OCT-1848}

Francisco Larco, natural de Santa Margherita (Rapallo), hijo de ... Larco y Angela Maria Sastreto (ITA), casado con Antonia Canevaro, natural de Zoagli (Génova), hija de Juan Bautista y Julia Canevaro. / Lib. 13, f. 201 /

071 / BAU. 05-ENE-1849

Federico Reinaldo Duboe, natural de Hamburgo, de 28 años, hijo de Eduardo Duboe y Julia Sans (ALE), apadrinado por Demetrio Olavegoya. /Lib. 23, f. 14/

072/ DEF. 09-FEB-1849

El coronel Salvador Soyer, natural de Bayona, marido de Mercedes Lavalle (FRA), enterrado en la iglesia de San Francisco.

\section{3/ MAT. 25-OCT-1849}

Andrés Denegri, natural de Laigueglia (Albenga), residente en Lima hace 4 años, hijo de Pedro Denegri y Catalina Frementi (ITA), casado con María Recarte, natural de Lima, hija de José Recarte y Josefa Carrillo. /Lib. 13, f. 93v/

\section{4/ MAT. 19-ENE-1850}

Hugo Roberto Francisco Jameson, natural de Londres, residente en Lima hace 2 años, hijo de Hugo Jameson y Catalina Wartington (ING), casado con Enriqueta Conroy, natural de Lima, hija de Tomás Conroy y Petronila Paula Enderica. / Lib. 13, f. $98 v /$

\section{5/ MAT. 01-MAR-1850}

Estanislao Pedro Osterling, natural de Estocolmo, residente en Lima hace más de 16 años, viudo de Josefa Clemencia Eguía (SUE), casado con Luisa Eguía, natural de Lima, hija de Hilario Eguía y María Ruiz Pimentel. /Lib. 14, f. 176/

076/ MAT. 11-MAY-1850

Eugenio María Baumann de Mety, capitán del ejército francés, natural de París, residente en Lima hace 7 meses, hijo de Pedro José y Mariana Baumann de Mety. $(F R A)$, casado con Pascuala García, natural de Supe (Chancay), residente en Lima hace 2 años, hija de Manuel Fructuoso García y Manuela Goytisolo. / Lib. 13, l. 106v/

077 / BAU. 06-JUL-1850

José Pastor Hague, natural de Ortrose, de 28 años, hijo de Juan y Alicia Hague (ESC), apadrinado por Jose Noble. /Lib. 23, f. 95v/ 
078/ MAT. 10-OC'T-1850

Luis Camogli, natural de Varazze (Savona), hijo de Jerónimo Camogli y Catalina Negrote (ITA), casado con Petronila Denegri, natural de Lima, hija de Pedro Denegri y Maria del Carmen Valega. /Lib. 13, f. 117v/

079/ MAT. 05-ABR-1850

Ernesto Lestonnat, natural de Burdeos, residente en Lima hace 18 meses, hijo de Juan Lestonnat y Aglae Taudin (FRA), casado con Francisca Casanova, natural de Barcelona, hija de Juan Casanova y Rosa Rovira. /Lib. 13, f. 129v/

080/ MAT. 23-JUN-1851

Juan Bautista Grellaud, natural de Saint-Médard-de-Guizieres (Gironda), residente en Lima hace 16 años, hijo de Juan Bautista Grellaud y Catalina Breton (FRA), casado con Celedonia Cortés, natural de Lima, hija de Santiago Cortés y Dolores Alvarez del Villar. /Lib. 13, f. 135v/

081/ MAT. 07-JUL-1851

Felipe Lavarello, natural de Génova, hijo de Juan Lavarello y Maria Cichero (ITA), casado con Carolina Merea, natural de Génova, hija de José Merea y Feliciana Pellegrini. / Lib. 13, f. 137v/

082/ MAT. 10-JUL-1851

Pedro Pescetto, natural de Albisola (Génova), hijo de Bartolomé Pescetto y Maria Delfino (ITA), casado con Juana Piacho, natural de Lima, hija de Tomás Piacho y Manuela Bravo. /Lib. 13, f. 141/

083/ BAU. 23-OCT-1851

Jorge Edmundo Solf, natural de Hamburgo, de 25 años, hijo de Juan Andrés Solf y Federica Cristina Sohlmann $(A L E)$, apadrinado por Antonio Joaquín Ramos, natural de Santiago de Chile (por poder). /Lib. 23, f. 172/

084/ MAT. 20-NOV-1851

Jorge Edmundo Solf, natural de Hamburgo (ALE), casado con María Natividad Sepúlveda, natural de Cañete, hija de José Antonio Sepúlveda y Gregoria Laguna. /Lib. 13, f. 151/

085/ MAT. 04-FEB-1852

Santiago Marcenaro, natural de Génova, residente en Lima hace 10 años, hijo de Andrés Marcenaro y Maria Viso $(I T A)$, casado con Catalina Sotomayor, natural de Lima, hija de Manuel Sotomayor y Carmen Ponciano. /Lib. 13, f. 157v/

086/ MAT. II-MAY-1852

Enrique Tremouille, natural de Burdeos, hijo de Juan Tremouille y Maria Bartel (FRA), casado con Marcelina Deustua, natural de Tacna, hija de Martín Deustua y Francisca Pomadera. /Lib. 13, f. 168v/

087 / MAT. 3()-MAY-18.52

Enrique Swayne, escocés, anglicano, hije de David Swavne y Cristina Wallace (ESC), casado con Lucia Virginia Mariátegui, natural de Lima, hija del Dr. Francisco Javier Mariátegui y Juana Palacio y Salas. /Lib, 14, I. 18v/ 
088/ MAT. 01-FEB-1853

Sebastián Celle, natural de Génova, hijo de Simón Celle y María Ferrari (ITA), casado con Francisca Musso, natural de Génova, hija de José Musso y María Canepa. /Lib. 13, f. 199/

089/ MAT. 05-FEB-1853

Bernardo Marrou, natural de Lafargues, hijo de Juan Marrou y María Lacase (FRA), casado con Eusebia Jiménez, natural de Matucana (Huarochirí), hija de Esteban Jiménez y Mariana Yauri. /Lib. 13, f. 194v/

090/ MAT. 23-FEB-1853

Pedro José Broggi, natural de Olivone (Tesino), hijo de José Broggi y María Escopun (SUI), casado con Baltasara Eloriaga, natural de Trujillo, hija -ilegítimade Antonio Eloriaga y Estefania Izquierdo. /Lib. 13, f. 198/

\section{1/ MAT. 21-JUL-1853}

Dr. Manuel Solari, natural de Génova (ITA), casado con Dominga Argumaniz, natural de Lima, hija de Francisco Argumaniz y María de la O Muñoz. /Lib. 14, f. $7 \mathrm{v} /$

\section{2/ MAT. 07-NOV-1853}

Nicolás Solari, natural de Chiavari (Génova), resldente en Lima hace 6 años, hijo de Agustín Solari y Martina Bartarelli (ITA), casado con Baltasara Fernandini, natural de Ica, residente en Lima hace 5 años, hija -ilegitima- de Félix Fernandini y Juana Sáenz. /Lib. 14, f. 18/

093/ MAT. 15-DIC-1853

Amador Dubois de Beauvegard, natural de Vannes (Morbihan), residente en Lima hace 2 años, hijo de Luis Dubois de Beauvegard y Clemencia de Caradeux $(F R A)$, casado con Carmen Peralta, natural de Tarija, resldente en Lima hace 7 años, hija de Casimiro Peralta y Trinidad Arce. /Lib. 14, f. 22/

094/ MAT. 23-ABR-1854

Leonardo Canessa, natural de Génova, residente en Lima hace 11 años, hijo de Benito Canessa y María Servino (ITA), casado con Teresa Lazo, natural de Guayaquil, residente en Lima hace más de 20 años, viuda de Matias Olmedo. /Lib. 14, f. 28v/

095/ MAT. 26-ABR-1854

Aquiles Courret, natural de Burdeos, hljo de Francisco Courret y Calixta Chalet $(F R A)$, casado con Carolina Albertini, natural de Versalles, hija de Maximiliano Albertini y Francisca Veyán y Arguedas. /Lib. 14, f. 29/

096/ MAT. 12-AGO-1854

Francisco Bryce, natural de Edimburgo, presbiteriano, hijo de Francisco Bryce y Juana Weddel (ESC), casado con Clementina López. Aldana, natural de Lima, hija del Dr. Fernando López. Aldana y Carmen Larriva. /Lib. 14, f. 68/ 
097/ BAU. 24-NOV-1854

Juan Manuel Cristián Kast, natural de Hannover, de 24 años, hijo de Jorge Federico Kast y Dorotea Scholle $(A L E)$, apadrinado por el Dr. Juan Mariano Cossio, vocal de la Corte Superior de Lima. /Lib. 24, f. 82 /

098/ MAT. 07-ENE-1855

Zacarias Elejalde, natural de Salinas (Guipúzcoa), residente en Lima hace 7 años, hijo de Julián Elejalde y Teresa Escurra (ESP), casado con Manuela Rosas, natural de Lima, hija de José Rosas y Juana Capetillo. /Lib. 14, f. 75v/

099/ MAT. 18-ENE-1855

Dr. Juan Copello, médico, natural de Chiavari (Génova), residente en Lima hace 9 años, viudo de Luisa Simonetti (ITA), casado con María Santos Rosas, natural de Lima, hija de Nicolás Rosas y María Rosario Barragán. /Lib. 14, f. 79/

100/ MAT. 10-SET-1855

Eduardo Soyer, natural de Burdeos, residente en Lima hace 21 años, hijo de Guillermo Soyer y Juana Trigant $(F R A)$, casado con Juana Serbigón, natural de Lima, hija -ilegítima- de Agustín Serbigón y Juana Nepomuceno N. /Lib. 14, f. $87 v /$

\section{$101 /$ DEF. 16-OCT-1855}

Enrique Mifflin, inglés, de 40 años, marido de Manuela Cavero (ING), enterrado en la iglesia de la Merced.

102/ MAT. 07-ENE-1856

Eduardo Nohesell, alemán, hijo de Felipe Nohesell y Margarita Helgenberg (ALE), casado con Juana Manuela Zúñiga, natural de Arequipa, de 24 años, hija -ilegítima-de Francisco Zúñiga y Luisa Manrique. /Lib. 14, f. 93v/

103/ MAT. 15-FEB-1858

José Salomón Bachmann, suizo, residente en Lima hace 6 años, hijo de Jacobo Bachmann y Dorotea Berl (SUI), casado con Josefa Amunátegui (o Ramos), natural de Lima, hija de padres no conocidos. /Lib. 14, f. 141v/

104/ MAT. 12-ABR-1858

Enrique Antonio Scheelje, natural de Oldemburgo, hijo de Federico Scheelje y Juana Sofía Cristina Zugener $(A L E)$, casado con María Vera, natural de Trujillo, hija de Antonio Vera y Martina Alvarado. /Lib. 14, f. 144v/

\section{5/ DEF. 21-MAY-1858}

Gaspar Palmieri, natural de Córcega, de 60 años, marido de María Valega (ITA), enterrado en la iglesia de San Francisco.

106/ DEF. 21 -JUL-1858

Manuel José Guillermo Walden, natural de Londres, de 50 años, marido de Juana Hoyos (ING), enterrado en la iglesia de los Huérfanos. 
107/ MAT. 07-ENE-1859

Luis Nosiglia, natural de Génova, residente en Lima hace 9 años, hijo de José Nosiglia y Maria Magdalena Grondona (ITA), casado con Catalina Corvetto, natural de Génova, hija de Manuel Corvetto y Luisa Buera. /Lib. 14, f. 155v/

108/ BAU. 24-FEB-1859

Carlos Kruger, natural de Altona (Holstein), de 29 años, hijo de Carlos Kruger y Elisa von Datelsyen $(A L E)$, apadrinado por el Dr. Manuel Antonio Colmenares. /Lib. 25, f. 111/

109/ MAT. 28-MAY-1859

Nicolás Nicolini, natural de Sahara (Génova), residente en Lima hace 9 años, hijo de Marcos Nicolini y Benedicta Chuguera (ITA), casado con Clorinda Dañino, natural de Lima, hija de Busión Dañino y Vicenta de la Puente. /Lib. 14, f. 162/

110/ DEF. 10-MAY-1860

Juan Bautista Grellaud, natural de Saint-Médard-de-Guizières (Gironda), de 46 años, marido de Celedonia Cortés, padre de seis hijos (FRA).

111 / MAT. 12-ABR-1861

Félix Dibós, francés, comerciante, mayor de 30 años, hijo de Félix Dibós y María Gracia Duplan (FRA), casado con Ernestina Pflücker, natural de Lima, hija de Julio Arnaldo Pflücker y Maria Manrique (o Taramona). /Lib. 14, f. 199/

\section{2/ MAT. 16-OCT-1861}

Juan Bautista Nicolini, italiano, viudo de Camila Solimano, hijo de Juan Bautista Nicolini y Margarita Azalini (ITA), casado con María Rivarola, natural de Génova, hija de Federico e Isabel Rivarola. /Lib. 14, f. 209v/

\section{3/ MAT. 31-DIC-1861}

Juan Luis Dammert, natural de Hamburgo, hijo de Juan Dammert y Guillermina Ansin $(A L E)$, casado con Juana Alarco, natural de Lima, hija de Julián Alarco y Mercedes Espinoza. /Lib. 14, f. 216v/

114/ MAT. 05-ABR-1862

Waldo Augusto Graña, natural de Galicia, hijo de José Antonio Graña y Clementina González (ESP), casado con Andrea Reyes, natural de Lima, hija de Andrés Reyes y Antonia Largacha. /Lib. 14, f. 219v/

115/ MAT. 18-AGO-1862

Augusto Dreyfus, francés, comerciante, hijo de Eduardo Dreyfus y Sara Gauraumon (FRA), casado con Sofía Bergmann, natural de Lima, hija de Federico Bergmann y Estanislada Rublo. /Lib. 14, f. 231v/

116/ MAT. 06-DIC-1862

Ricardo Higgin Hartley, inglés, hijo de Benjamín Hartley y Leonor Higgin (ING), casado con Miguelina Sara de Sousa Ferreyra, natural de Lima, hija de Antonio de Sousa Ferreyra y Paula Gutiérrez de Caviedes. /Lib. 14, f. 237/ 
117/ MAT. 08-DIC-1862

Nicolás Young, sueco, hijo de Juan Benito Young y Simona Frenson (SUE), casado con María Candelaria Osambela, natural de Lima, hija de José Osambela y Gabriela Muncilla. /Lib. 14, f. 233v/

118/ MAT. 30-JUL-1863

Eduardo Nugent, irlandés, hijo de Tomás Nugent y Maria Fenell (IRL), casado con Carolina Gonzales, natural de Lima, hija de José María Gonzales y Tránsito Ganas. /Lib. 14, f. 245/

119/ MAT. 01-OCT-1863

Pablo Fernando Sattler, alemán, protestante, hijo de Pablo Sigismundo Sattler y Carlota Dorotea Amalia Beste $(A L E)$, casado con Maria Beatriz Pflücker, natural de Lima, hija de Julio Arnaldo Pflücker y María Manrique (o Taramona). /Lib. 14, f. 249v/

\section{0/ MAT. 20-OCT-1863}

Pedro Larrabure, francés, hijo de Andrés Larrabure y Maria Apatay (FRA), casado con Juana Othéguy, francesa, hija de Juan Othéguy y María Echegaray Borda. /Lib. 14, f. 250v/

121/ BAU. 27-ENE-1864

Juan Bautista MacLeod, escocés, de 27 años, hijo de Jorge y María MacLeod (ESC), apadrinado por Antonio Alarcón. /Lib. 25, f. 514/

\section{2/ MAT. 10-MAR-1864}

Carlos Prince, natural de París, de 29 años, hijo de Juan Carlos Prince y Luisa Lescher (FRA) casado con Josefa Bengoa, natural de Alca (La Unión), de 20 años, hija -ilegítima- de Jacinto Bengoa y María Mogrovejo. /Lib. 23, f. 58/

\section{3/ MAT. 05-NOV-1864}

Prudencio Maertens, belga, hijo de Luis Maertens y Rosalía Geiseis (BEL), casado con Alejandrina Bastard, francesa, hija de Pablo Bastard y Victoria Kuer. / Lib. 15, f. $10 /$

\section{4/ MAT. 03-DIC-1864}

Juan Antonio Migliorati, marqués de Migliorati, ministro plenipotenciario de Italia, natural de Génova, hijo del marqués Juan Bautista Migliorati y Essila Baiggi (ITA), casado con María Clorinda Canevaro, natural de Lima, hija de José Francisco Canevaro, cónsul general de Italia, y Francisca Zenona Valega. /Lib. 15, f. $22 \mathrm{v} /$

\section{5/ DEF. 21-MAR-1865}

Estanislao Pedro Osterling, natural de Estocolmo, de 75 años, marido de Luisa Eguía $(S U E)$, muerto de hidropesia.

126/ MAT. 07-DIC-1865

Eduardo Raymundo Lacroix, francés, hijo de Santiago Lacroix y Juana Dorotea 
Domecq (FRA), casado con Isabel de Aliaga y Puente, natural de Lima, hija de Juan de Aliaga y Manuela de la Puente. /Lib. 15, f. 36/

127/ MAT. 14-ENE-1866

Claudio Rebagliati, natural de Noli (Savona), hijo de Angel Rebagliati y Lilla Ricardoni (ITA), casado con Florinda Raybaud, natural de Lima, hija de Luis Raybaud y Manuela Salazar. /Lib. 15, f. 37v/

128/ MAT. 02-JUL-1866

Juan Bromley, norteamericano, viudo de Mariana Gardin (USA), casado con Manuela Berninzon, hija de Agustín Berninzon y Manuela Molina. /Lib. 15, f. 49/

129/ MAT. 10-NOV-1867

Gabino de Menchaca, natural de Algorta (Vizcaya), comerciante, de 29 años, hijo de Juan António de Menchaca y María Vicenta Manene (ESP), casado con Carolina Figari, natural de Lima, hlja de Juan Figari y Eulalia Rosas. /Llb. 15, f. $98 /$

\section{0/ MAT. 10-NOV-1867}

Bernardo Emilio Fort, francés, comerciante, de 26 años, hijo de Miguel Fort y Rosa Veguerie (FRA), casado con María Dolores Figari, natural de Lima, hija de Juan Figari y Eulalia Rosas. /Lib. 15, f. 98v/

\section{1/ MAT. 22-NOV-1867}

Saúl Laurie, polaco, hijo de David Laurie y Rosa Manoschewitz (POL), casado con Rosalía Rolando, natural de Lima, hija de Francisco Rolando y Dominga Rodriguez Silva. /Lib. 15, f. 99v/

132/ DEF. 30-MAR-1868

Pedro José Broggi, natural de Olivone (Tesino), de 54 años, marido de Baltasara Eloriaga (SUI).

\section{3/ BAU. 14-JUN-1868}

Ernesto Federico Basilio Roedel, alemán, comerciante, de 32 años, hijo de Juan Adolfo Roedel y Cristina Egerland $(A L E)$, apadrinado por Agustin Ramón Hurtado. /Lib. 26, f. 485/

134/ DEF. 19-SET-1868

Samuel Stanhope Prevost, norteamericano, comerciante, de 60 años, marido de Maria Mauricia Moreyra, padre de cinco hijos (USA), muerto de parálisis.

135/ DEF. 27-MAR-1869

Juan Cristián Brenner, alemán, de 53 años, marido de Regina Brenner, padre de cuatro hijos (ALE).

136/ MAT. 22-JUL-1869

Juan Gildemeister, natural de Bremen, protestante, de 46 años, hijo de Martín Gildemeister y Luisa Ebers $(A L E)$, casado con Manuela Prado, natural de Lamba- 
veque, de 28 años, hija de José Manuel Prado y Petronila Jimenè. /Lib. 15, f. $154 /$

137/ MAT. 04-DIC-1869

Santiago Magill, natural de Belfast, viudo de Juana Molero (IRL), casado con Rosario Villacampa, natural de Lima, hija de Pedro Villacampa y Juliana Viana. /Lib. 15, f. 169/

138/ DEF. 01-MAR-1870

José Pastor Hague, natural de Ortrose, boticario, de 48 años, marido de Virginia Gignoux, con sucesión (ESC).

139/ DEF. 27-ABR-1870

Enrique Berckemeyer, alemán, secretario de la legación de Bolivia, de 56 años, marido de Josefa Bernales, padre de cuatro hijos $(A L E)$, muerto de hipetrofia, intestado.

140/ MAT. 29-JUN-1870

Julio Hugo Hoefken, alemán, hljo de Julio Hoefken y Matilde Weeren (ALE), casado con Virginia Carrillo, natural de Lima, de 26 años, viuda, hija de Julián Carrillo y Ascencia Meneses. /Lib. 15, f. 188v/

141 / DEF. 03-SET-1870

Juan Dartnell, natural de Newcastle (Down), vicecónsul de Gran Bretaña en Pisco, marido de Mercedes Carlota Guise, padre de tres hijas (IRL), muerto de apoplejía cerebral, en la isla Guañape (La Libertad), el 21-AGO-1870.

142/ DEF. 19-SET-1870

Eduardo Raymundo Lacroix, francés, marido de Isabel de Aliaga y Puente, padre de dos hijos (FRA), muerto en Panamá el 02-SET-1870.

143/ DEF. 04-FEB-1871

José Salomón Bachmann, suizo, agricultor, de 36 años, marido de Josefa Amunátegui (o Ramos), padre de cinco hijos (SUI).

144/ DEF. 21 -FEB-1871

Be'rnardo Poumarroux, natural de Burdeos, comerciante, de 67 años, padre de cuatro hijos (FRA).

145/ DEF. 29-MAR-1871

Federico Augusto Matias Elmore, natural de Londres, de 65 años, marido de Josefa Fernández de Córdova, padre de nueve hijos (INC).

146/ DEF. 28-JUN-1871

Pedro Denegri, italiano, de 64 años, viudo de Marta del Carmen Valega, padre de siete hijos (ITA). 
147/ DEF. 30-SET-1871

Dr. Juan Gallagher, irlandés, de 70 años, marido de Isabel Hamilton Robertson, padre de cinco hijos (IRL), muerto de asesinato.

148/ MAT. 23-DIC-1871

Cristián Krüger, alemán, protestante, de 28 años, hijo de Federico Krüger y Ana Kellermann ( $A L E$ ), casado con Elvira Derteano, natural de Lima, de 16 años, hija -ilegitima- de Dionisio Derteano y Juana Saavedra. /Lib. 16, f. Iv/

149/ MAT. 03-FEB-1872

Eugenio Courret, natural de Burdeos, fotógrafo, de 32 años, hijo de Francisco Courret y Calixta Chalet (FRA), casado con Emllia Baserre, francesa, de 21 años, hija de Emilio Federico Baserre y Luisa Cortade. /Lib. 17, f. 180/

150/ MAT. 14-SET-1872

Carlos Renardo Pflücker, natural de Waldenburg (Silesia), protestante, viudo de Gertrudis Rico, hijo de Bernardo Pflücker y Leonora Schmiedel (ALE), casado con Paula Ampuero. /Lib. 16, f. 18/

151/ MAT. 18-ENE-1873

Augusto Dreyfus, francés, comerciante, viudo de Sofía Bergmann (FRA), casado con Luisa María Gonzales, natural de Trujillo, de 25 años, hija de Vicente Gonzales Pinillos y Emilia de Orbegoso Pinillos. /Lib. 16, f. 27v/

152/ MAT. 29-MAY-1873

Juan Pedro Julio Fort, francés, de 27 años, hijo de Miguel Fort y Rosa Veguerie (FRA), casado con Clorinda Toribia Figari, natural de Lima, de 19 años, hija de Juan Figari y Eulalia Rosas. /Lib. 16, f. 40/

\section{3/ DEF. 14-JUN-1873}

José Vanderalmey, francés, comerciante, de 50 años, hijo de Enrique y Catalina Vanderalmey, soltero (FRA).

154/ MAT. 02-AGO-1873

Eduardo Carlos Dubois, natural de Poughkeepsie (Nueva York), de 40 años, hijo de Cornelio Dubois y Julia Moore (USA), casado con Manuela Emilia Gonzales, natural de Trujillo, de 24 años, hija de Vicente Gonzales Pinillos y Emilia de Orbegoso Pinillos. /Lib. 16, f. 44/

155/ MAT. 04-DIC-1875

Claudio Rebagliati, natural de Noli (Savona), de 32 años, viudo de Florinda Raybaud (ITA), casado con Raquel Carbajal, natural de Arequipa, de 19 años, hija del comisario ordenador Mariano Carbajal y Joaquina Chávez. / Lib. 16, f. 103/

156/ MAT. 12-FEB-1876

Adhemar Montagne, natural de Douai (Nord), de 43 años, viudo, hijo de Adhemar Montagne y Adela Créquin ( $F R A)$, casado con María Eugenia Markholz, inglesa, de 32 años, hija de Carlos Federico Markholz y Eugenia Walsh. /Lib. 16, l. 105/ 
157/ MAT. 25-MAY-1876

Oscar Antonio Federico Augusto Heeren, natural de Hamburgo, de 36 años (ALE), casado con Ignacia Barreda y Osma, natural de Lima, de 28 años, hija de Felipe Barreda y Aguilar y Carmen de Osma y Ramírez de Arellano. /Lib. 16, 1. $116 \mathrm{v} /$

158/ BAU. 05-DIC-1876

Rodolfo Luis Holtig, natural de Hamburgo, de 28 años, hijo de Eduardo Augusto Holtig y Maria Luisa Precht (ALE), apadrinado por Gustavo Muente. /Lib. 29, f. $373 /$

\section{9/ MAT. 22-AGO-1877}

Tomás Garvasi, italiano, de 44 años, hijo de Jose Garvasi y Fidela Trinsa (ITA), casado con Juana Denegri, natural de la China, de 30 años, hija de padres no conocidos. /Lib. 16, f. 137 /

\section{0/ DEF. 10-FEB-1878}

Luis Camogli, natural de Varazze (Savona), de 55 años, marido de Petronila Denegri, padre de tres hijos (ITA), muerto en la hacienda Bocanegra (Lima), cogido por la rueda de una máquina. /Lib. 20, f. 111 /

161/ MAT. 17-MAR-1878

Francisco Talleri, suizo, de 29 años, hijo de Angel Talleri y Angela Soldini (SUI), casado con Angela Raineri, natural de Lima, de 19 años, hija de Guillermo Raineri y Enriqueta Lund. /Lib. 17, f. 4/

162/ DEF. 20-ABR-1878

Jorge Chamot, alemán, de 60 años, viudo, padre de un hijo (ALE), muerto de mal de orina. /Lib. 20, f. 132/

\section{3/ MAT. 30-ABR-1878}

Juan Alberto Labrousse, francés, de 26 años, hijo de Juan Labrousse y María Mercier $(F R A)$, casado con Francisca Luzzani, suiza, de 28 años, hija de Jacobo Luzzani y Francisca Lugino. /Lib. 17, 1. 3/

164/ MAT. 21-AGO-1879

Minor Keith Meiggs, norteamericano, de 26 años, hijo de Enrique Meiggs y Carolina Dayle (USA), casado con Emilia Matilde Rossignol, natural de Lima, de 24 años, hija de Oscar Rossignol y Matilde Sánchez. /Lib. 17, f. 137/

165/ DEF. 20-DIC-1879

Adolfo D. Harismendy, francés, de 48 años, marido de Carmı́n Figari, padre de dos hijos (FRA), muerto de afección al corazón. / Lib. 20, l. 336 /

166/ MAT. 31-JUL-1880

Carlos Jorge Antonio Ludowieg, natural de Schnackenburg (Luneburgo), luterano, de 32 años, hijo de Carlos Cristián Ludowieg y Solia Dorotea Schmidt (ALE), casado con Frecia Juana Cantuarias, de 31 años, hija de Tiburcio Cantuarias y Rafacla Flores. /Lib. 17, f. 279/ 
167/ DEF. 22-ENE-1881

Santiago Frassinetti, natural de Supia de Arena, zapatero, de 57 años, marido de Petronila Lamalle (ITA), muerto de grano de peste. /Lib. 21, f. 107/

168/ DEF. 05-JUL-1881

Enrique D. Chandler, norteamericano, empresario, de 50 años, casado (USA), muerto de disentería. /Lib. 21, f. 157/

169/ MAT. 21-JUL-1881

Hermann Naldemar Schröder, natural de Hamburgo, luterano, de 30 años, hijo de Cristián Matías Schröder y Matilde Troost (ALE), casado con Isabel Mendoza, natural de Lima, de 19 años, hija de Manuel Mendoza e Isabel Barreda. /Lib. 17, f. $189 /$

\section{0/ MAT. 24-AGO-1881}

Julio Ludowieg, natural de Schnackenburg (Luneburgo), luterano, de 39 años, hijo de Carlos Cristián Ludowleg y Sofía Dorotea Schmidt (ALE), casado con Juana Rafaela Cantuarias, natural del Callao, de 20 años, hija de Tiburcio Cantuarias y Georgina Stanley. /Lib. 17, f. 189/

171 / DEF. 15-ABR-1882

Juan Magot, francés, comerciante, de 33 años, hijo de Juan Magot y Mariana Pradin, casado (FRA), muerto de tuberculosis. /Lib. 21, f. 219/

\section{2/ MAT. 22-JUN-1883}

Pablo Macera, natural de Milán, de 35 años, hijo de Octavio Macera y Mónica Semino (ITA), casado con Elisa Frassinetti, natural de Lima, de 23 años, hija de Santiago Frassinetti y Petronila Lamalle. /Lib. 17, f. 262/

\section{3/ MAT. 05-SET-1883}

Agustín Guérin, francés, de 43 años, hijo de Antonio Guérin y Catalina Vasserst (FRA), casado con Carmen Figari, natural de Lima, de 38 años, viuda de Adolfo D. Harismendy, hija de Juan Figari y Eulalia Rosas. /Lib. 18, f. 77/

\section{4/ BAU. 29-FEB-1884}

Enrique Jorge Hemmerde, natural de Londres, hijo de Jaime Godofredo Hemmerde y Francisca Pape (ING), apadrinado por Guillermo Corthorn. /Lib. 31, f. $182 /$

\section{5/ MAT. 15-MAY-1884}

Enrique Jorge Hemmerde, natural de Londres, de 25 años (ING), casado con María Esther Vera Pérez de Tudela, natural de Lima, de 22 años, hija de Casimiro Vera Pérez de Tudela y María Eugenia Taramona. /Lib. 17, f. 205/

\section{6/ DEF. 24-OCT-1884}

Bernardo Emilio Fort, francés, comerciante, de 43 años, marido de María Dolores Figari, padre de diez hijos (FRA), muerto de apoplejia fulminante. /Lib. 20, f. $414 /$ 


\section{7/ MAT. 29-NOV-1884}

Pablo Carriquiry, francés, de 36 años, hijo de Francisco Carriquiry y Juana Charot (FRA), casado con Inés Larrabure, natural de Lima, de 18 años, hija de Pedro Larrabure y Juana Othéguy. /Lib. 17, f. 216/

178/ MAT. 19-MAR-1886

José Giacometti, italiano, hijo de Luis Giacometti y la condesa Teresa Marzono de Visconti (ITA), casado con Victoria Soyer, hija de Simón Soyer y Josefina Morote. /Lib. 17, f. 233/

179/ MAT. 17-AGO-1889

Dr. Walter Stubbs, natural de Santo Tomás (Islas Virgenes), residente en Lima hace 14 años, protestante, de 35 años, hijo de Enrique Ricardo y Carolina Augusta Stubbs (DIN), casado con María Catalina Perron, natural de París, viuda de Francisco La Roche. /Lib. 17, f. 302/

\section{0/ MAT. 31-ENE-1891}

Alejandro León Truel, francés, de 35 años, hijo de Emilio Truel y Paulina Thinot $(F R A)$, casado con Margarita Larrabure, natural de Lima, de 17 años, hija de Pedro Larrabure y Juana Othéguy. /Lib. 17, f. 330/

\section{1/ MAT. 05-MAR-1891}

Herbert C. Tweddle, norteamericano, de 27 años, hijo de Herbert W. C. Tweddle y Maria Robinson (USA), casado con Consuelo Valdeavellano, natural de Lima, de 21 años, hija de Benito Valdeavellano y Juana Canaval. /Lib. 17, f. 330/

182/ BAU. 30-AGO-1891

Reginald Seymour Gubbins, irlandés, de 29 años, hijo de Guillermo Gubbins y María Russell (IRL). /Lib. 32, f. 111 /

183/ MAT. 12-SET-1891

Reginald Seymour Gubbins, irlandés, de 29 años (IRL), casado con Rosa María Pastor, natural de Lima, de 24 años, hija de Juan Pastor y Rosa Raborg. / Lib. 17, f. $340 /$

184/ MAT. 18-OCT-1891

Juan Guibovich, austriaco, de 27 años, hijo de Lucas y Ana Guibovich (AUS), casado con Angélica Mondéjar, natural de Lima, de 16 años, hija de Manuel Mondéjar y Tomasa Sánchez. /Lib. 18, f. 148v/

185/ DEF. 04-NOV-1892

Enrique Witt, alemán, cónsul de Dinamarca, de 93 años, viudo de María Sierra (ALE), muerto de consunción. /Lib. 23, f. 96/

186/ MAT. 27-NOV-1892

Herbert C. Tweddle, norteamericano, de 28 años, viudo de Consuelo Valdeavellano (USA), casado con Juana Valdeavellano, natural de Lima, de 19 años, hija de Benito Valdeavellano y Juana Canaval. /Lib. 17, f. 376/ 
187/ DEF. 02-MAR-1899

Ernesto Malinowski, polaco, ingeniero, de 80 años, soltero (P(OL), muerto de ataque cerebral. / Lib. 23, f. 400/

188/ DEF. 09-OCT-1899

Jorge Deacon, norteamerlcano, de 80 años, marido de Rosa Tovar (USA), muerto de fiebre perniciosa. / Lib. 23, f. 424/

189/ BAU. 14-JUL-1901

Julio Luis Rachitoff, natural de Odesa (Ucrania), de 17 años, hijo de Miguel Rachitoff y Paulina Goldenhorn (RUS). /Lib. 35, f. 376/

190/ DEF. 04-FEB-1904

Gustavo Augusto Heudebert, natural de París, de 69 años, marido de María J. Gonzales (FRA), muerto de parálisis. /Lib. 24, f. 40v/

191/ DEF. 04-ABR-1906

Federico Uranga, español, de 50 años, casado (ESP), muerto de ataque cerebral. /Lib. 24, f. 93/

\section{2/ DEF. 13-JUN-1911}

Adolfo Westphalen, alemán, de 71 años, hijo de Enrique y ... Westphalen (ALE), muerto de afección al corazón. /Lib. 26, f. 76/

193/ DEF. 23-OCT-1915

Andrés Gaillour, francés, de 66 años, hijo de Bernardo Gaillour y Feliciana Larrabure, casado (FRA), muerto de gripe. /Lib. 26, f. 289/

194/ MAT. ca. 1810?

El barón Timoteo de Nordenflicht, natural de Mitau (Curlandia), consejero íntimo del rey de Polonia, director general de la comisión de minas del virreinato (POL), casado con María Josefa Cortés y Azúa, natural de Santiago de Chile.

195/ MAT. ca. 1815?

Andrés Bolognesi, natural de Génova (ITA), casado con Juana Cervantes Pacheco, natural de Arequipa.

196/ MAT. ca. 1826?

El general de brigada Clemente de Althaus, natural de París, oficial del ejército prusiano (ALE), casado con Manuela Flores del Campo, natural de Arequipa.

197/ MAT. ca. 1832?

Pedro Denegri, italiano, hijo de Pedro Denegri y Angela Vasallo (ITA), casado con Maria del Carmen Valega, natural de Lima, hija de Félix Valega y María Josefa Iribar.

198/ MAT. ca. 1835?

Carlos Renardo Pflücker, natural de Waldenburg (Silesia) (ALE), casado con 
Gertrudis Rico, natural de Guayaquil, hija del coronel ... Rico y Francisca Rocafuerte.

199/ MAT. ca. 1848?

Geraldo Garland, natural de York, hijo de Ricardo Garland y Maria Myers (ING), casado con Enriqueta von Lotten, natural de Arequipa, hija de Antonio von Lotten y Maria Sierra y Velarde.

200/ MAT. ca. 1858?

Carlos Herybery, natural de Breslau (Silesia) (ALE), casado con Ventura Maldonado, natural de Pasco.

201/ MAT. ca. 1860?

Carlos Kruger, natural de Altona (Holstein) $(A L E)$, casado con Rosaura Correa, natural de Lima, hija del general Cirilo Correa y Garay y Maria Luisa Boza y Carrillo de Albornoz.

202/ MAT. ca. 1860?

Francisco Nicacio de Igartua, natural de Plencia (Vizcaya), comerciante, hljo de José Antonio de Igartua y Francisca de Egusquiza (ESP), casado con Juana Francisca Andraca, natural de Lima, hija de Juan Bautista Andraca y Francisca Busolo.

203/ MAT. ca. 1862?

Juan Carlos Federico Blume, ingeniero de Estado, natural de Santo Tomás (Islas Virgenes) (DIN), casado con Enriqueta Corbacho, natural de Lima, hija de José Maria Corbacho y Abril y María Manuela Tirado y Coronel Zegarra.

204/ MAT. ca. 1862?

José Julián Loredo, natural de Vizcaya, comerciante, hijo de Juan A. Loredo y Marla Revilla (ESP), casado con Beatriz Román, natural de Lima, hija de Rafael Román y Manuela Vidal.

205/ MAT. ca. 1865?

Juan Federico Guillermo Hohagen, ingeniero de Estado, natural de Prusia (ALE), casado con Escolástica Farfán, natural del Cuzco.

206/ MAT. ca. 1872?

Francisco Luis Crosby, natural de Nantucket (Massachusetts) (USA), casado con Mercedes Tizón, natural del Callao.

207/ MAT. ca. 1875?

Miguel Trefogli, ingeniero de Estado, suizo (SUI), casado con Cora Francisca Garcla, natural de Lima.

208/ MAT. ca. 1880?

Juan Dietrich Buse, natural de Bremen (ALE), casado con Laura Antallo, natural de Lima. 
209/ MAT. ca. 1880?

Dr. Christian Dam, natural de Santa Cruz (Islas Virgenes) (DIN), casado con Nisida Durán, natural de Lima.

210/ MAT. ca.1895?

Samuel Brahm, natural de Hamburgo (ALE), casado con Adelaida Clotilde Schreitmüller, natural de Lambayeque.

\section{INDICE DE APELLIDOS EXTRANJEROS}

Althaus (ALE), 196.

Bachmann (SUI), 103, 143.

Baglietto (ITA), 64.

Baumann de Metz (FRA), 76.

Berckemeyer (ALE), 139.

Blume (DIN), 203.

Boler (ING), 2.

Bolognesi (ITA), 195.

Bracklow (DIN), 63.

Brahm (ALE), 210.

Branch (FRA), 9.

Brandon (ING), 62.

Brenner (ALE), 135.

Broggi (SUI), 90, 132.

Bromley (USA), 128.

Browne (ING), 24.

Bryce (ESC), 96.

Buse (ALE), 208.

Camogli (ITA), 78, 160.

Canessa (ITA) 94.

Canevaro (ITA), 31, 70, 124.

Carriquiry (FRA), 177.

Celle (ITA), 88.

Conroy (IRL), 18, 74.

Copello (ITA), 99.

Courrejolles (FRA), 60.

Courret tFRA), 95, 149.

Crosby (USA), 206.

Chamot (ALE), 162.

Chandler (USA), 168.

D'Brot (FRA), 53.

Dam (DIN), 209.

Dammert (ALE), 113.

Dartnell (IRL), 68, 141.

Deacon (USA), 188.

Denegri (ITA), 73, 78, 146, 197.
Deplace (FRA), 49.

Dibós (FRA), 111.

Dinegro (ITA), 29.

Dorival (FRA), 45.

Dreyfus (FRA), 115, 151.

Duboe (ALE), 71.

Dubois (USA), 154.

Dubois de Beauvegard (FRA), 93.

Elejalde (ESP), 98.

Elmore (ING), 33, 145.

Flint (USA), 35.

Fort (FRA), 130, 152, 176.

Frassinetti (ITA), 167, 172.

Gaillour (FRA), 193.

Gallagher (IRL), 147.

Gambini (ITA), 6.

Garland (ING), 199.

Garvasi (ITA), 159.

George (ALE), 44.

Giacometti (ITA), 178.

Gildemeister (ALE), 136.

Gillo (GRE), 13.

Ginocchio (ITA), 14, 69.

Graña (ESP), 114.

Grellaud (FRA), 80, 110.

Gubbins (IRL), 182, 183.

Guérin (FRA), 173.

Guibovich (AUS), 184.

Guichard (FRA), 43.

Guise (ING), 15, 20, 68.

Hague (ESC), 77, 138.

Harismendy (FRA), 165.

Hartley (ING), 116.

Heeren (ALE), 157.

Hemmerde (ING), 174, 175.

Herybery (ALE), 200. 
Heudebert (FRA), 190.

Himpseen (ALF), 66.

Hocfken (ALE), 140.

Hohagen (ALE), 205.

Holtig (ALE), 158.

Igartua (ESP), 202.

Jameson (ING), 74.

Kast (ALE), 97.

Kelly (IRL), 12.

Kruger (ALE), 108, 201.

Kruger (FRA), 55.

Krüger (ALE), 148.

Labrousse (FRA), 163.

Lacroix (FRA), 126, 142.

Lanfranco (ESP), 61.

Larco (ITA), 70.

Larrabure (FRA), 120, 177, 180.

Laurie (POL), 131.

Lavarello (ITA), 81.

Lembcke (ALE), 40, 41.

Lestonnat (FRA), 79.

Loredo (ESP), 204.

Lorente (ESP), 57.

Ludowieg (ALE), 166, 170.

MacKay (ESC), 28.

MacLeod (ESC), 121.

Macera (ITA), 172.

Maertens (BEL), 123.

Magill (IRL), 59, 137.

Magot (FRA), 171.

Malinowski (POL), 187.

Marcenaro (ITA), 85.

Marrou (FRA), 89.

Massa (ITA), 16.

Meiggs (USA), 164.

Menchaca (ESP), 129.

Mifflin (ING), 101.

Mlgliorati (ITA), 124.

Migone (ITA), 50.

Montagne (FRA), 156.

Montané (FRA), 36.

Nicolini (ITA), 109, 112.

Nobelich (ALE), 26, 27.

Nohesell (ALE), 102.

Nordenflicht (POL), 194.

Nosiglia (ITA), 107.

Nugent (IRL), 118.

O'Brien (IRL), 37.

Oboussier (BEL), 21.
Osma (ESP), 1.

Osterling (SUE), 19, 67, 75, 12.5.

Palmieri (ITA), 105.

Pescetto (ITA), 82.

Pflücker (ALE), 38, 39, 111, 119, 150,

198.

Polack (ITA), 34.

Poumarroux (FRA), 144.

Prevost (USA), 47, 134.

Prince (FRA), 122.

Rachitoff (RUS), 189.

Radcliff (USA), 22, 56.

Rebagliati (ITA), 127, 155.

Risso (ITA), 17.

Roedel (ALE), 133.

Rouaud (FRA), 58.

Rousson (FRA), 11.

Saberbein (HUN), 32.

Sacio (ITA), 3, 7, 48.

Sattler (ALE), 119.

Scheelje (ALE), 104.

Schröder (ALE), 169.

Smith (ING), 10.

Solari (ITA), 91, 92.

Solf (ALE), 83, 84.

Sousa Ferreyra (POR), 8, 116.

Soyer (FRA), 25, 72, 100.

Stubbs (DIN), 179.

Stuber (ALE), 54.

Suito (ITA), 46, 69.

Swayne (ESC), 87.

Talleri (SUI), 161.

Tenaud (FRA), 52.

Thorne (USA), 51.

Trefogli (SUI), 207.

Tremouille (FRA), 86.

Truel (FRA), 180.

Tweddle (USA), 181, 186.

Umbert (ESP), 23.

Uranga (ESP), 191.

Valega (ITA), 4, 29, 31, 197.

Vallarino (ITA), 65.

Vanderalmey (FRA), 153.

Venzano (ITA), 5.

Walden (ING), 42, 106.

Westphalen (ALE), 192.

Winder (ING), 30.

Witt (ALE), 185.

Young (SUE), 117. 